\title{
Appendizitisdiagnostik mit der Temposchwelle
}

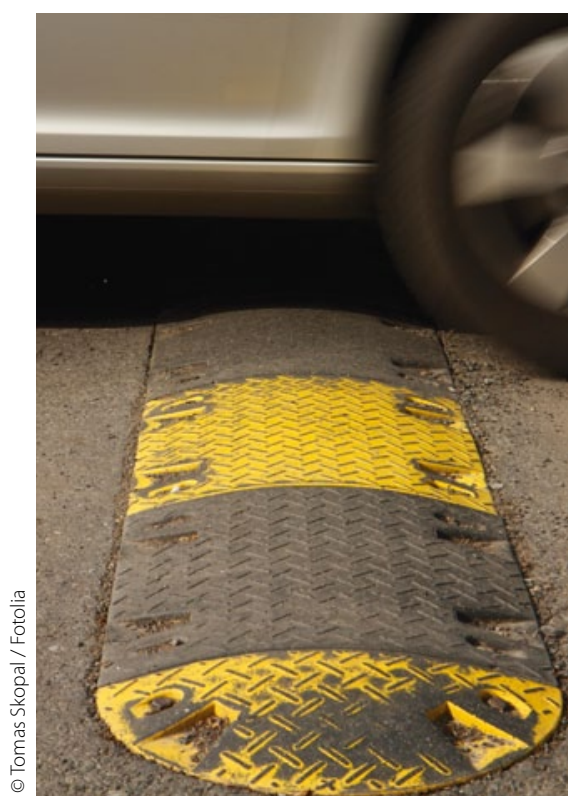

Wer diese Schwelle schmerzfrei überfährt, hat keine Appendizitis.

Der Schmerz, den ein Patient mit Appendizitisverdacht im Krankenwagen erfährt, wenn dieser über eine Schwelle fährt, ist mindestens so zuverlässig wie die üblichen klinischen Zeichen.

- Die akute Appendizitis ist nicht nur der häufigste bauchchirurgische Notfall, sondern gehört auch zu den am häufigsten fehldiagnostizierten Erkrankungen, was nach Literaturangaben $\mathrm{zu}$ negativen Appendektomieraten zwischen $5 \%$ und $42 \%$ führt. Vor allem in den frühen Stadien ist die Diagnose klinisch nicht leicht zu stellen.

Beim Transport ins Krankenhaus haben Patienten mit Appendizitis immer wieder berichtet, dass sie beim Fahren über Temposchwellen besondere Schmerzen empfunden hätten. Diese Beobachtungen wurden nun in einer prospektiven Studie an 101 Patienten mit Verdacht auf Appendizitis im Alter zwischen 17 und 76 Jahren in einem englischen Krankenhaus systematisch untersucht. 64 Patienten waren mit dem Krankenwagen über die Temposchwel- len am Eingang des Krankenhauses gefahren. Bei 34 wurde eine akute Appendizitis histologisch bestätigt. 33 dieser Patienten berichteten über Schmerzen beim Passieren der Temposchwellen. Die Sensitivität des Tests errechnete sich mit hervorragenden 97\%, die Spezifität ist naturgemäß deutlich niedriger und betrug nur $30 \%$.

Mit einem positiven prädiktiven Wert von $61 \%$ und einem negativen prädiktiven Wert von $90 \%$ muss sich der Test nicht hinter klinischen und bildgebenden Verfahren verstecken. Der Wahrscheinlichkeitsquotient für ein positives Testergebnis lag bei 1,4 , für ein negatives Ergebnis bei 0,1. Der Temposchwellentest hatte eine bessere Sensitivität und negativen Wahrscheinlichkeitsquotienten als klinische Zeichen wie Wandern der Schmerzen oder Loslassschmerz. Durch die hohe Sensitivität eignet sich der Temposchwellentest vor allem zur Ausschlussdiagnose einer Appendizitis oder anderer Erkrankungen des Abdomens. Die geringe Spezifität bedeutet allerdings, dass viele Patienten. die Schmerzen beim Passieren von Temposchwellen empfinden, nicht notwendigerweise eine Appendizitis haben müssen.

\section{Kommentar}

Temposchwellen sind somit nicht nur zur Verhütung von Unfällen geeignet, sondern leisten auch einen wichtigen Beitrag zur medizinischen Diagnostik. Allerdings muss man die Patienten auch nach dem Phänomen fragen. Höchste Zeit, dass dieser Test in Lehrbücher der Anamnese und der Untersuchung aufgenommen wird. Umgekehrt sollten vor keinem Krankenhaus Temposchwellen fehlen.

- H. F. Ashdown et al. (Korres.: helen.ashdown@phc.ox.ac.uk): Pain over speed bumps in diagnosis of acute appendicitis: diagnostic accuracy study. BMJ 2012; 345: e8012 